\title{
L'organisation religieuse catholique et le droit des sociétés : l'exemple de la paroisse
}

Sandie Lacroix-De Sousa

\section{(2) OpenEdition}

\section{Journals}

Édition électronique

URL : http://journals.openedition.org/rdr/391

DOI : $10.4000 /$ rdr.391

ISSN : 2534-7462

\section{Éditeur}

Presses universitaires de Strasbourg

Édition imprimée

Date de publication : 24 avril 2018

Pagination : $39-53$

ISBN : 979-10-344-0017-1

ISSN : 2493-8637

\section{Référence électronique}

Sandie Lacroix-De Sousa, «L'organisation religieuse catholique et le droit des sociétés : l'exemple de la paroisse », Revue du droit des religions [En ligne], 5 | 2018, mis en ligne le 23 octobre 2019, consulté le 19 novembre 2020. URL : http://journals.openedition.org/rdr/391 ; DOI : https://doi.org/10.4000/rdr. 391

La revue du droit des religions est mise à disposition selon les termes de la Creative Commons Attribution - Pas d'Utilisation Commerciale 4.0 International - CC BY-NC 4.0. 


\section{L'ORGANISATION RELIGIEUSE CATHOLIQUE ET LE DROIT DESSOCIÉTÉS:L'EXEMPLEDELAPAROISSE}

\section{Sandie LACROIX-DE SOUSA}

Université d'Orléans, Centre de recherche juridique Pothier

\section{RÉSUMÉ}

$\mathrm{Au}$ sein de l'organisation religieuse catholique, la paroisse constitue la «cellule de base» réunissant une communauté déterminée de fidèles confiée à la charge pastorale d'un curé. Structurée selon les dispositions du Code de droit canonique, la paroisse organise une répartition des pouvoirs entre des instances dirigeantes, consultatives et de contrôle. Pour mener à bien sa mission pastorale, elle jouit de certains revenus, assume des charges, se soumet aux obligations comptables et rappelle manifestement l'organisation et le fonctionnement d'une société. Pour autant, ce rapprochement demeure relatif, la paroisse ne réunit pas les éléments constitutifs d'une société et ne saurait juridiquement bénéficier de cette qualification.

\section{Abstract}

Within the Catholic organization, the parish constitutes the "basic cell", bringing together a community of believers, entrusted to the pastoral charge of a priest. Structured according to the provisions of the Code of Canon Law, the parish organizes a division of powers between governing, consultative and supervisory bodies. In order to carry out its pastoral mission, it benefits from certain incomes, assumes responsibilities, is subject to accounting obligations and clearly recalls the organization and functioning of a company. However, this comparison remains relative, the parish does not unite the constituent elements of a company and thus cannot legally benefit from this qualification. 
T a religion catholique et le droit des sociétés relèvent a priori de deux L secteurs formellement antagonistes. L'une s'intéresse aux relations de l'homme avec la puissance divine, l'autre répond à des préoccupations essentiellement patrimoniales et financières. Certains évoquent même, au sujet des relations entre l'Église et l'entreprise, des rapports d'opposition voire

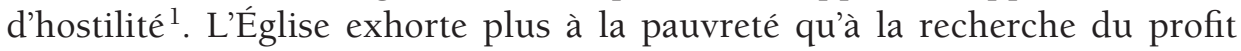
pécuniaire. À plusieurs reprises, les Évangiles rappellent d'ailleurs la méfiance de Jésus envers la richesse - «nul ne peut servir Dieu et l'argent ${ }^{2}{ }$ - ou relatent encore sa colère lorsqu'il chasse les marchands du temple ${ }^{3}$. En pratique, l'Église a néanmoins besoin d'argent pour faire vivre ses institutions et ses relations avec le commerce animent traditionnellement des débats passionnés ${ }^{4}$. La perception d'une Église riche et économiquement puissante est encore très présente aujourd'hui. Sont régulièrement dénoncés - comme mythes ou réalités - les trésors immobiliers de l'institution, la gestion opaque des dons et des legs ou encore la tarification indirecte des sacrements ${ }^{5}$. L'objet de la présente étude dédiée aux relations entre l'organisation religieuse catholique et le droit des sociétés en droit français ne consiste pas à traiter une nouvelle fois le thème de l'Église et de l'argent ${ }^{6}$, ni même à procéder à une analyse comptable ou financière de l'institution. À l'évidence, le droit des sociétés ne se confond pas avec le droit comptable ou le droit financier. Il occupe une place particulière au sein du «droit des affaires» applicable aux entreprises et aux activités économiques ${ }^{7}$ et réunit plus spécifiquement les règles applicables à toutes les sociétés qu'elles soient civiles ou commerciales, de personnes ou de capitaux, dotées de la personnalité morale ou non ${ }^{8}$.

Souvent rattaché au droit des groupements qui réunit notamment les règles applicables aux groupements de l'économie sociale et solidaire comme les

1. M. VASSEUR, «L'entreprise et l'argent», D. 1982, chron., p. 11.

2. Évangile selon saint Matthieu 6,24-34.

3. Évangile selon saint Matthieu 21,12-13.

4. J. CARbonnier, «La religion, fondement du droit? Droit et Religion», Arch. phil. dr. 1993, p. 98; S. JAHEL, «Droit des affaires et religions», RID comp. 2001, p. 879.

5. F. Mayaux., «L'Église et l'argent. La perception des Français, entre mythes et réalités», Études 2003/12, p. 643.

6. E. Perrot, «L'argent sous les feux de la religion», Arch. phil. dr. 1997, p. 61: il existerait une «connaturalité entre l'institution religieuse et l'institution monétaire [...] qui se manifeste par une concurrence explicite entre argent et religion».

7. J. Hilaire, «Quel droit des affaires? Essai de prospective juridique: le droit des affaires», in Mélanges A. Sayag, Paris, Litec, 1997, p. 148; M. CABRILlaC, «Vers la disparition du droit commercial? », in Mélanges J. Foyer, Paris, PUF, 1997, p. 329.

8. M. Cozian, A. Viandier et F. Deboissy, Droit des sociétés, Paris, LexisNexis, $29^{e}$ éd. 2016, $\mathrm{n}^{\circ} 3$ et s.; D. Vidal et K. Luciano, Cours de droit général des sociétés, Paris, Lextenso, $2015, \mathrm{n}^{\circ} 2$. 
associations ou les fondations ${ }^{9}$, le droit des sociétés régit les différentes étapes de la vie sociale ainsi que la situation des différents acteurs qui l'animent. Trouvant essentiellement sa source dans les dispositions du Code civil, du Code de commerce et du Code monétaire et financier, il met en place un certain nombre de principes structurant la société parmi lesquels figurent entre autres: la répartition des pouvoirs entre différents organes, le respect de l'intérêt social, l'autonomie de la personne morale, la règle majoritaire, l'appréhension des situations de conflits d'intérêts. En ce sens, le droit des sociétés s'inscrit manifestement dans la catégorie des sciences d'organisation $^{10}$. Dépassant le débat classique sur la nature de la société - contrat ou institution - on considère d'ailleurs aujourd'hui que la société est surtout un modèle de contrat-organisation dans lequel les intérêts des parties convergent pour atteindre un même but ${ }^{11}$. Technique d'organisation, la société se distingue de la notion économique d'«entreprise» qui désigne plus largement la réunion de moyens matériels et humains permettant de se livrer à une activité économique autonome ${ }^{12}$. La qualification juridique de société obéit à des conditions cumulatives précises issues de l'article 1832 du Code civil: la mise en commun d'apports, la vocation aux résultats et l'affectio societatis. Juridiquement, «entreprise» et «société» ne se confondent donc pas. Il existe des entreprises qui ne sont pas exploitées par une société ${ }^{13}$ et, à l'inverse, certaines sociétés, utilisées comme technique d'organisation du patrimoine, ne coiffent aucune entreprise ${ }^{14}$.

Les notions d' «entreprise " et de "société» ne sont pas étrangères à l'Église, mais celle-ci ne les distingue pas comme le font les juristes. Quelle que soit la structure juridique choisie, l'encyclique Centesimus annus écrite par Jean-Paul II en 1991 invite à définir l'entreprise comme une société

9. M.-H. Monserie-Bon, L. Grosclaude, C.-M. Benard, Droit des sociétés et des groupements, Paris, Lextenso, 2016.

10. J. Paillusseau, «La logique organisationnelle dans le droit. L'exemple du droit des sociétés», in Mélanges Béguin, Paris, Litec, 2005, p. 567.

11. En ce sens P. Didier, «Le consentement sans l'échange: contrat de société», RJ com. 1995, p. 75; «Brèves notes sur le contrat-organisation», in Mélanges François Terré, Paris, Dalloz, 1999, p. 636: «le contrat organisation est un contrat qui de manière explicite ou implicite, définit une tâche, la divise en parties élémentaires et les répartit, d'une manière ou d'une autre, entre les contractants.»

12. M. Pedamon et H. Kenfack, Droit commercial, Paris, Dalloz, $4^{e}$ éd. 2015, n 432; B. MerCADAL, «La notion d'entreprise», in Mélanges Jean Derruppé, Paris, Litec, 1991, p. 9; P. Didier, «Une définition de l'entreprise», in Études offertes à Pierre Catala, Paris, Litec, 2001, p. 849.

13. C'est le cas de l'entreprise individuelle ainsi que de l'entreprise individuelle à responsabilité limitée.

14. V. par ex., les sociétés civiles immobilières de gestion. 
de personnes: «l'entreprise ne peut être considérée seulement comme une société de capital; elle est en même temps une société de personnes dans laquelle entrent, de différentes manières et avec des responsabilités spécifiques, ceux qui fournissent le capital nécessaire à son activité et ceux qui y collaborent par le travail ${ }^{15}$.» L'objectif est d'insister sur l'attention qui doit toujours être portée à l'environnement humain. Faisant fi de la séparation classique entre sociétés de personnes - dans lesquelles les associés répondent personnellement des dettes de la société - et sociétés de capitaux - dans lesquelles le risque assumé par les associés se limite au montant des apports l'encyclique rappelle que la société représente avant tout une communauté de personnes mettant ensemble leur capital ou leur travail pour le bien de toute la société. Son but «n'est pas uniquement de faire du profit mais l'existence même d'une communauté de personnes qui, de différentes manières, recherchent la satisfaction de leurs besoins fondamentaux ${ }^{16} »$.

Dans la structure hiérarchique mise en place par l'Église catholique se trouvent même des groupements rassemblant une communauté de fidèles et placés sous l'autorité de l'évêque, soumis au droit des sociétés et des groupements. Selon les Évangiles, c'est aux apôtres qu'avait été confié le gouvernement de l'Église sous l'autorité de Pierre. Dans le diocèse, l'évêque gouverne, sous l'autorité du pape «la portion du peuple qui lui est confiée $e^{17}$ ». Cette articulation se nomme la collégialité. La prééminence de l'évêque se retrouve patente dans l'organisation et le fonctionnement de l'association diocésaine. Instituée depuis le modus vivendi de 1924 établi entre le SaintSiège et l'État français pour pallier le refus des catholiques de constituer des associations cultuelles ${ }^{18}$, elle est instaurée dans chaque diocèse pour «subvenir aux frais et à l'entretien du culte catholique, sous l'autorité de l'évêque en communion avec le Saint-Siège et conformément à la constitution de l'Église catholique ${ }^{19} »$. Soumise à la fois au droit canonique et au droit civil, elle doit se conformer à des règles particulières en matière de composition, de fonctionnement, de gestion financière, de dissolution. Les

15. Encyclique Centesimus annus, 43.

16. Ibid., 35 .

17. Selon le décret conciliaire Christus Dominus sur la charge pastorale des évêques, signé le 28 octobre 1965 par Paul VI; P. Valdrini, J. Vernay, J.-P. Durand et O. Echappé, Droit canonique, Paris, Dalloz, 1989, $\mathrm{n}^{\circ} 202$.

18. F. Renaud, Les associations diocésaines, Étude sur le statut de l'Église en France, Paris, Dunod, 1923; F. Messner, P.-H. Prelot et J.-M. Woehrling (dir.), Traité de droit français des religions, Paris, Litec, 2003, n 1623 et s.; B. BASDEVANT-GAUDEMET, « Droit et religions en France», RID comp. 1998, p. 346.

19. Statuts types, article $2 \S 1$. 
termes «statuts», "mandat», «gouvernance», «conseil d'administration», «assemblée générale» figurent ainsi formellement dans le champ lexical de l'organisation religieuse et le régime juridique de l'association diocésaine est même référencé dans un célèbre répertoire de droit des sociétés ${ }^{20}$.

Le diocèse n'est cependant pas l'unique circonscription de l'organisation du culte catholique et le Code de droit canonique prévoit précisément que « tout diocèse ou toute autre Église particulière est divisée en parties distinctes ou paroisses ${ }^{21} »$. La paroisse offre à chacun des fidèles la possibilité de conduire quotidiennement sa vie de chrétien. Le droit canonique la définit comme «la communauté précise de fidèles qui est constituée d'une manière stable dans l'Église particulière et dont la charge pastorale est confiée au curé, comme à son pasteur propre, sous l'autorité de l'évêque diocésain ${ }^{22} »$. C'est en son sein que se déroulent l'administration des sacrements et les célébrations liturgiques ordinaires, d'où sa qualification de «première communauté concernée par l'organisation de la vie religieuse ${ }^{23}$ » ou encore de "véritable cellule de base de l'organisation diocésaine ${ }^{24} »$. Pour que le culte puisse se dérouler de manière harmonieuse et conformément au principe hiérarchique, la paroisse obéit à des règles d'organisation et de gestion précisément instaurées par le droit canonique. Elle demeure néanmoins ignorée du droit civil et a fortiori du droit des sociétés et des groupements.

Inexistant du point de vue du droit français, le statut des paroisses en droit civil interroge toutefois. Réunissant une communauté de fidèles animés par une même volonté de faire vivre un projet commun, organisant une répartition des pouvoirs entre différents acteurs chargés d'œuvrer ensemble à la réalisation de la mission pastorale, jouissant de certains revenus et assumant corrélativement certaines dépenses, la paroisse n'est pas sans rappeler les traits caractéristiques d'une société. Lorsqu'on les interroge, certains curés admettent même qu'ils se sentent parfois à la tête d'une petite ou moyenne entreprise en raison des nombreuses tâches de gestionnaire qu'ils assument. Il apparaît dès lors plus pertinent de sonder les relations entre l'organisation religieuse catholique et le droit des sociétés à l'échelon de la

20. A. Bougnoux, «Associations soumises à un régime spécial - Associations cultuelles. Associations diocésaines », JCl. Sociétés Traitée, fasc. 175-50, nº 96.

21. Canon 374.

22. Canon $515 \S 1$.

23. B. Basdevant-Gaudemet, Histoire du droit canonique et des institutions de l'Église latine, $X V^{e}-X X^{e}$ siècle, Paris, Economica, 2014, $n^{\circ} 384$.

24. J. PALARD, «Processus de transformation d'une organisation religieuse», ASSR, n 60/1, 1985, p. 138. 
paroisse, précisément. Le droit des sociétés ne s'appliquant pas aux seuls groupements bénéficiant de la personnalité morale peut-il régir les relations des différents acteurs de la paroisse? Les créanciers de la paroisse pourraient avoir intérêt à obtenir la requalification de leur débiteur en société; ce statut leur permettrait de poursuivre personnellement le curé ou les fidèles pour le recouvrement de leurs créances. En droit civil, la paroisse peut-elle prétendre à la qualification de société?

À l'examen, il apparaît que la paroisse évoque manifestement certaines règles caractéristiques du droit des sociétés dans son fonctionnement (1), mais qu'elle ne réunit pas les conditions juridiques nécessaires pour une qualification en société (2).

\section{LA PAROISSE ÉVOQUANT LE DROIT DES SOCIÉTÉS DANS SON FONCTIONNEMENT}

Comme en droit des sociétés, le droit canonique prévoit au sein de la paroisse une répartition des pouvoirs entre différents organes exerçant leurs attributions conformément à un intérêt supérieur (1.1) et selon des règles de gestion formelles (1.2).

\subsection{LA GOUVERNANCE DE LA PAROISSE}

À l'instar d'une société dans laquelle les pouvoirs sont répartis entre le dirigeant qui représente la société et l'assemblée générale des associés qui vote les décisions stratégiques, la paroisse distingue clairement les pouvoirs de représentation et d'administration du curé et les attributions consultatives et délibératives du conseil paroissial pour les affaires économiques.

\subsubsection{LE CURÉ}

Le curé, dit pasteur propre de la paroisse qui lui a été confiée, exerce la charge pastorale de la communautée ${ }^{25}$. Les textes le désignent comme un représentant de l'évêque dans la paroisse ayant mission d'exercer pour une communauté de fidèles déterminée les trois fonctions de l'Église d'enseignement,

25. Th. BLot, Le curé, pasteur: des origines à la fin du xxe siècle. Étude historique et juridique, Paris, Téqui, 2000; A. Borras, «La notion juridique de curé dans le Code de droit canonique», $R D$ canon., $\mathrm{n}^{\circ} 27,1987$, p. 215. 
de sanctification et de gouvernement. Comme un dirigeant de société, le curé assume ainsi des fonctions de représentation, d'administration et de direction: «Dans toutes les affaires juridiques, le curé représente la paroisse selon le droit; il veillera à l'administration des biens de la paroisse ${ }^{26}$.» Il est également assujetti à des règles précises de nomination et de révocation. Nommé par l'évêque, le curé ne peut être transféré à un autre office que par une procédure spécifique obligeant l'évêque à proposer par écrit sa décision et laissant à l'intéressé la possibilité de refuser. La procédure de révocation prévue en cas de "perte de la bonne estime ou de l'aversion de la part des paroissiens envers leur curé», de "mauvaise administration des biens temporels» ou de «grave détriment ou trouble grave apportés à la communion ecclésiale ${ }^{27} »$ rappelle encore les notions de «loyauté», de «juste motif de révocation» ou de «faute de gestion» bien connues du droit des sociétés.

La nomination possible de vicaires paroissiaux comme coopérateurs du curé de la paroisse ressemble encore au mécanisme de la délégation de pouvoirs très utilisé en droit des sociétés ${ }^{28}$. Selon le droit canonique, les vicaires collaborent à l'ensemble du ministère d'une paroisse ou pour des charges plus précises territoriales ou personnelles. L'étendue de leur charge est déterminée notamment par la lettre de nomination de l'évêque qui peut augmenter ou modifier leurs droits et devoirs ainsi que par le curé luimême ${ }^{29}$. Le contrat de mandat sur lequel repose la délégation de pouvoir en droit des sociétés semble servir ici de modèle; l'organe dirigeant transfère une partie de ses pouvoirs à un fondé de pouvoir qui dispose pleinement de la compétence, de l'autorité et des moyens nécessaires pour accomplir la mission qui lui est confiée.

Des règles de non-cumul des mandats s'appliquent également au curé. Il est par exemple prévu qu'un curé n'a la charge paroissiale que d'une seule paroisse ${ }^{30}$. À titre dérogatoire, notamment pour prendre en compte les difficultés d'organisation d'un diocèse en cas de pénurie de prêtres, il est possible de prévoir que plusieurs paroisses voisines soient confiées à un même curé. Un système de cogérance peut aussi être mis en place. «Là où

26. Canon 532.

27. Canon 1741.

28. N. Ferrier, La délégation de pouvoir, technique d'organisation de l'entreprise, Paris, LexisNexis, 2005; J.-M. Moulin et S. Colliot, «La vie des délégations de pouvoirs dans l'entreprise», BJS 2012, n 398, p. 745; A. Larcena, A. ElinEaU, «Pratique des délégations de pouvoirs: règles et évolutions récentes de cet outil de répartition des pouvoirs et responsabilités», Dr. sociétés 2012, étude 4.

29. P. Valdrini, J. Vernay, J.-P. Durand et O. Echappe, op. cit., nº 257.

30. Canon 526. 
les circonstances l'exigent ${ }^{31} »$, il est possible qu'une paroisse ou plusieurs paroisses soient prises en charge par une équipe de prêtres exerçant la charge pastorale solidairement, c'est-à-dire sans hiérarchie entre eux. Dans ce cas, l'un d'entre eux est nommé modérateur de l'exercice de la charge pastorale, il dirige l'activité commune et en répond devant l'évêque ${ }^{32}$.

\subsubsection{LE CONSEIL PAROISSIAL POUR LES AFFAIRES ÉCONOMIQUES}

Le Code de droit canonique fait une obligation à tout administrateur de biens d'Église d'être assisté dans sa tâche par un conseil ${ }^{33}$. Dans la paroisse, le curé est ainsi obligatoirement assisté d'un conseil paroissial pour les affaires économiques: "Il y aura dans chaque paroisse le conseil pour les affaires économiques qui sera régi, en plus du droit universel, par les règles que l'évêque diocésain aura portées; dans ce conseil, des fidèles, choisis selon ces règles, apporteront leur aide au curé pour l'administration des biens de la paroisse $^{34}[\ldots] »$. Organe collégial, ce conseil est un lieu de coopération des fidèles qui, avec le curé, assurent la conservation et l'entretien du patrimoine paroissial; ses attributions peuvent être rapprochées de celles de l'assemblée générale des associés.

Composé de fidèles nommés pour un mandat de cinq ans renouvelable, le conseil paroissial pour les affaires économiques assure plusieurs missions essentielles au bon fonctionnement de la paroisse ${ }^{35}$. En pratique, il établit le budget prévisionnel en étudiant les investissements possibles en fonction des recettes et des dépenses prévues. Il veille à la bonne organisation du denier de l'Église. Il suit les comptes et les approuve en fin d'année comme le fait l'assemblée générale des associés après clôture des comptes annuels de société. Il peut arriver que son consentement soit expressément requis; par exemple, en cas d'aliénation d'immeubles, en cas d'acte d'administration extraordinaire ou pour des travaux supérieurs à certains montants ${ }^{36}$. De

31. Canon 517.

32. P. Valdrini, J. Vernay, J.-P. Durand et O. Echappe op. cit., n 266.

33. Le canon 536 prévoit encore la désignation facultative d'un conseil pastoral paroissial présidé par le curé et réunissant des personnes représentatives des divers secteurs de la vie paroissiale.

34. Canon 537.

35. L. Serée De Roch, Administration et fiscalité des biens d'Église, Perpignan, Artège, 2012, p. 41.

36. ArchevêChé de Paris. Direction générale des affaires éConomiques, Ordonnance modifiant les statuts des conseils paroissiaux pour les affaires économiques: http://www.paris.catholique. fr/Textes-de-reference-sur-les.html [consulté le 17 janv. 2018]. 
manière générale, il aide le curé dans sa prise de décision, par ses recherches, ses avis et définit les orientations pour la gestion des revenus et profits des biens d'Église; cette mission rappelle celle de l'assemblée générale des associés qui choisit les orientations concernant la stratégie de la société. Le conseil pour les affaires économiques se réunit au minimum deux fois par an: pour voter le budget et pour contrôler sa réalisation. À la fin de chaque séance, un compte rendu est établi - sorte de procès-verbal d'assemblée - et communiqué à l'économe diocésain. Les comptes rendus et les ordres du jour sont ensuite archivés par année au secrétariat paroissial.

\subsection{LES RÈGLES DE GESTION DE LA PAROISSE}

Les principales règles comptables et les procédures de contrôle qui s'appliquent aux sociétés s'appliquent également à la paroisse.

\subsubsection{LES RÈGLES COMPTABLES}

La paroisse a une mission pastorale mais elle doit également assurer les ressources indispensables pour organiser le culte divin, supporter les charges concernant les frais nécessaires aux célébrations cultuelles, comme les assurances des biens, les déplacements, le chauffage, l'électricité des immeubles dont elle a la charge. Comme pour les groupes de sociétés, la technique employée est celle de la consolidation des comptes: les comptes enregistrés au niveau des paroisses sont agrégés aux comptes de l'association diocésaine qui seule a la personnalité morale. Au sein de chaque paroisse existent donc des services de comptabilité pour la saisie comptable, l'enregistrement chronologique et la conservation des données. Les comptes de l'association diocésaine suivent ensuite le même dispositif que celui mis en place pour les sociétés: publicité au Journal officiel et certification par un commissaire aux comptes ${ }^{37}$. La mise en place de ces procédures comptables vise à assurer la fiabilité des comptes annuels. Comme souvent en droit des sociétés, la transparence financière est au cœur des préoccupations.

Sur le plan pratique, la présentation des comptes annuels de la paroisse doit répondre à un formalisme précis. Conformément au droit comptable, doivent être établis: le bilan qui fournit un état de la situation active et passive du patrimoine de la paroisse à une date donnée; le compte de résultat

37. Ordonnance $\mathrm{n}^{\circ} 2005-856$ du 28 juillet 2005, modifiant l'article 4 de la loi $\mathrm{n}^{\circ} 87-571 \mathrm{du}$ 23 juillet 1987 sur le développement du mécénat. 
qui récapitule les produits et les charges de l'année; l'annexe qui complète et commente ces informations ${ }^{38}$. Un dossier permanent contenant les documents contractuels de la paroisse est tenu et conservé par le comptable de la paroisse. Il permet au curé de prendre rapidement connaissance de l'état patrimonial de sa nouvelle affectation et contribue ainsi à une meilleure gestion. Il répertorie entre autres les biens immobiliers et mobiliers, les contrats souscrits par la paroisse, la liste des salariés et leurs statuts.

\subsubsection{LES RÈGLES DE CONTRÔLE}

Plusieurs règles de contrôle sont mises en place dans la paroisse pour garantir la qualité de la gestion. Il convient par exemple de respecter la règle de séparation des pouvoirs au sein du conseil paroissial pour les affaires économiques. Les fonctions de direction, de trésorier et de comptable doivent ainsi relever de trois personnes différentes. En pratique, la fonction de direction exercée par le curé l'autorise à commander les fournitures et à percevoir les recettes; la fonction de trésorier payeur permet de régler les factures et de déposer en banque les recettes; la fonction de comptable consiste à enregistrer et saisir les dépenses et recettes.

Surtout, à l'instar d'un dirigeant de société, le curé doit respecter les pouvoirs attribués aux autres organes. Pour accomplir certains actes, il doit obtenir l'autorisation préalable de l'économe diocésain, du conseil diocésain pour les affaires économiques ou encore de l'évêque. Les membres du conseil diocésain disposent effectivement d'un droit de regard sur les comptes de la paroisse et doivent être consultés pour l'embauche de personnel, les dépenses dépassant certains seuils ou toute acquisition ou aliénation de biens et particulièrement de biens immeubles ${ }^{39}$. L'autorisation de l'évêque est requise pour toute vente ou acquisition d'un bien, pour toute embauche, pour toute dépense importante envisagée par le curé, mais après avis du conseil diocésain pour les affaires économiques. Les seuils sont fixés par la Conférence des évêques de France ${ }^{40}$.

Afin d'éviter les situations de conflits d'intérêts que le droit des sociétés tend pareillement à appréhender, le droit canonique rend incompatible avec

38. C. com, art. L. 123-12, al. 3.

39. Canon 493: « [...] il revient au conseil pour les affaires économiques de préparer chaque année, selon les indications de l'évêque diocésain, le budget des recettes et des dépenses à prévoir pour le gouvernement du diocèse tout entier pour l'année à venir ainsi que d'approuver les comptes des recettes et des dépenses pour l'année écoulée. »

40. Canons $1276 \S 2$ et $1281 \S 1$ et $\S 2$. 
le mandat de conseiller paroissial pour les affaires économiques la parenté avec le curé, le fait d'être salarié de la paroisse ou du prêtre et certaines fonctions publiques importantes tel que conseiller général ou maire ${ }^{41}$.

Sans nul doute, le fonctionnement de la paroisse tel qu'établi par le droit canonique rappelle certains principes directeurs structurant la société: une distribution des pouvoirs de gouvernance, de consultation et de contrôle entre différents organes obéissant à des règles de gestion comparables et chargés d'agir dans le respect d'un intérêt supérieur qui les supplante. Effectivement, de même que dirigeants et associés doivent agir dans l'intérêt de la sociétét ${ }^{42}$, curé et fidèles doivent ouvrer dans le souci de la dimension pastorale de la paroisse. Pour autant, le rapprochement entre paroisse et société connaît des limites et il ne saurait influer sur la qualification de la paroisse en droit civil.

\section{LA PAROISSE EXCLUE DU DROIT DES SOCIÉTÉS PAR SA QUALIFICATION JURIDIQUE}

En droit civil, la paroisse ne jouit pas de la personnalité morale (2.1) et ne réunit pas les conditions nécessaires à une qualification en société créée de fait (2.2).

\subsection{L'ABSENCE DE PERSONNALITÉ MORALE EN DROIT CIVIL}

Seul le droit canonique reconnaît à la paroisse la qualité de personne morale; au regard du droit civil, elle demeure un simple établissement de l'association diocésaine.

\subsubsection{LA PAROISSE, PERSONNE MORALE EN DROIT CANONIQUE}

Le droit canonique connaît la notion de personnalité morale ${ }^{43}$. Plus précisément, il distingue parmi les personnes morales celles qui existent en vertu du droit divin - l'Église catholique et le Siège apostolique de l'évêque de Rome - et les autres, créées par une disposition du droit ecclésiastique ${ }^{44}$.

41. Canon $492 \& 3$.

42. C. civ., art. 1848.

43. P. Gillet, La personnalité juridique en droit ecclésial, spécialement chez les décretistes et les décrétalistes et dans le Code de droit canonique, Malines, W. Godenne, 1927.

44. Canons 113 et 114 . 
Sont ainsi constitués en personnes juridiques des ensembles de personnes ou de choses ordonnés à une fin qui s'accorde avec la mission de l'Église et dépasse les intérêts des individus ${ }^{45}$.

S'agissant de la paroisse, le canon 515 alinéa 3 prévoit explicitement que «la paroisse légitimement érigée jouit de plein droit de la personnalité juridique». Au regard du droit canonique, elle est donc une personne juridique publique constituée d'un ensemble de fidèles. Il s'agit néanmoins d'un ensemble de personnes non collégial ${ }^{46}$ puisque juridiquement, l'action de la paroisse n'est pas déterminée par ses membres mais par le curé, pasteur propre de la communauté, qui n'est pas un membre comme les autres pour le gouvernement de la paroisse.

\subsubsection{LA PAROISSE, ÉTABLISSEMENT DE L'ASSOCIATION DIOCÉSAINE EN DROIT CIVIL}

Au regard du droit civil, la paroisse n'est pas dotée de la personnalité morale. Elle est considérée comme un établissement particulier de l'association civile diocésaine qui, seule, se voit reconnaître la personnalité juridique. L'association diocésaine bénéficie ainsi de la grande capacité et peut recevoir des legs ou encore émettre des reçus fiscaux. La constitution d'une telle association donne à l'Église le moyen légal de posséder un patrimoine et de récupérer certains biens. Un jugement remarqué du tribunal civil de Montauban en date du 7 juillet $1948^{47}$ a ainsi attribué à une association diocésaine un legs qui, initialement, avait été fait à une paroisse.

À l'analyse, il semble que l'Église ait souhaité «éviter une démultiplication de structures civiles plus ou moins autonomes pouvant faire perdre de vue aux fidèles la vocation spécifique des structures canoniques et leur unité $e^{48}$. Curé et fidèles n'ont pas la charge de faire exister juridiquement la paroisse en tant que sujet de droit autonome en créant par exemple une association paroissiale de type loi du $1^{\text {er }}$ juillet 1901.

45. Canon 114.

46. Canon $115 \& 2$.

47. T. civ. Montauban, 7 juill. 1948, D. 1948, p. 563; A. PONSARD, «Sur quelques difficultés relatives à l'interprétation des testaments. Le rôle du juge dans la détermination du légataire ou du bénéficiaire d'une charge», JCP G 1957, I, 1385.

48. En ce sens, P. Valdrini, J. Vernay, J.-P. Durand et O. Echappe op. cit., n ${ }^{\circ} 882$. 


\subsection{LA QUALIFICATION IMPOSSIBLE EN SOCIÉTÉ CRÉÉE DE FAIT}

Le droit des sociétés ne s'applique pas seulement aux sociétés dotées de la personnalité morale mais s'impose également aux «sociétés qui s'ignorent» ou sociétés créées de fait. Ne réunissant pas les conditions issues de l'article 1832 du Code civil, la paroisse ne saurait juridiquement bénéficier de cette qualification.

\subsubsection{LA NOTION DE SOCIÉTÉ CRÉÉE DE FAIT}

Les sociétés immatriculées au registre du commerce et des sociétés ne sont pas les seules sociétés reconnues juridiquement. Le droit admet également l'existence de sociétés non pourvues de personnalité morale à l'instar des sociétés en participation, des sociétés de fait ou des sociétés créées de fait ${ }^{49}$. Dans ces dernières, les parties n'ont pas conscience d'avoir créé entre elles une société et c'est a posteriori que le juge identifie les conditions constitutives et caractéristiques du contrat de société issues de l'article 1832 du Code civil: la mise en commun d'apports, la vocation aux bénéfices et aux pertes et l'affectio societatis.

Un créancier pourrait voir un intérêt à obtenir la qualification de la paroisse en société créée de fait. Au lieu de bénéficier d'une action contre ce seul établissement de l'association diocésaine, il pourrait valablement poursuivre, lorsque certaines conditions sont remplies, le curé ou des fidèles. Conformément aux articles 1871 et suivants du Code civil, les associés d'une société créée de fait sont effectivement engagés à l'égard des tiers dans les mêmes conditions que les associés d'une société en participation, c'est-à-dire solidairement si la société a un objet commercial et conjointement si son objet est civil. L'action du créancier est néanmoins subordonnée à la preuve de l'existence de la société créée de fait.

\subsubsection{LE DÉFAUT DES ÉLÉMENTS CONSTITUTIFS D'UNE SOCIÉTÉ}

Selon le Code civil, la preuve de l'existence d'une société créée de fait peut être apportée par tous moyens ${ }^{50}$. La jurisprudence adopte cependant, depuis plusieurs années, une approche particulièrement rigoureuse de ces

49. G. Kessler, "Société créée de fait: les leçons du droit comparé», D. 2005, p. 86; F.-X. LuCAS, «La société dite "créée de fait" », in Mélanges Y. Guyon, Paris, Dalloz, 2003, p. 737.

50. C. civ., art. 1871. 
sociétés non personnifiées ${ }^{51}$. Elle exige que les éléments cumulatifs caractérisant la société soient établis séparément sans qu'ils puissent «se déduire les uns des autres ${ }^{52} »$. Pour que la paroisse soit qualifiée juridiquement de société créée de fait, il conviendrait de prouver distinctement que les fidèles réalisent des apports, qu'ils sont animés d'une affectio societatis et qu'ils ont vocation aux bénéfices et aux pertes. À l'examen, une telle qualification semble peu probable.

S'agissant des apports réalisés par les fidèles, on peut songer à une requalification des dons ou legs faits au bénéfice de la paroisse pour l'identification d'apports en nature ou en numéraire ou aux compétences, au savoir-faire, à l'expérience mise au profit de la structure pour la caractérisation d'apport en industrie. Toutefois, le raisonnement implique de faire abstraction de l'intention libérale caractéristique des libéralités qui singularise manifestement la contribution apportée par chacun des fidèles catholiques à la paroisse, ce qui n'est pas juridiquement satisfaisant.

Concernant la preuve de l'affectio societatis, élément "psychologique ${ }^{53}$ » du contrat de société, il conviendrait de démontrer que les fidèles sont effectivement animés d'une "volonté commune de collaborer ensemble sur un pied d'égalité au succès de l'entreprise commune ${ }^{54} »$. Or, la jurisprudence appréhende de manière rigoureuse cette notion contestée du droit des sociétés, parfois qualifiée de «fuyante ${ }^{55}$ » ou d'«insaisissable $\mathrm{d}^{56} »$. À l'occasion de décisions rendues sur la reconnaissance d'une société créée de fait entre concubins, et dans un mouvement d'objectivation ${ }^{57}$, la Cour de cassation a ainsi distingué la volonté commune de s'associer dans un projet sociétaire, c'est-à-dire «l'intention de s'associer» et les motivations subjectives qui poussent des individus à collaborer, c'est-à-dire «la mise en commun

51. Pendant un temps, la Cour de cassation a caractérisé très facilement leur existence: Cass. req., 14 mars 1927: DH 1927, p. 223.

52. Cass. $1^{\text {re }}$ civ., 20 janv. 2010: Rev. sociétés 2010, p. 430, note B. Dondero; Cass. com., 23 juin 2004: Rev. sociétés 2005, p. 131, note F.-X. LuCAS; Cass. com., 3 avr. 2012: Dr. sociétés 2012, comm. 117, note R. MORTIER.

53. I. Tchotourian, Vers une définition de l'affectio societatis lors de la constitution d'une société, Paris, LGDJ, 2011; V. CuISINIER, L'affectio societatis, Paris, Litec, 2008; S. LACROIXDe Sousa, «Le rayonnement de l'affectio societatis», Rev. sociétés 2016, p. 499.

54. Cass. com., 3 juin 1986: Rev. sociétés 1986, p. 585, note Y. GuYon.

55. M. Despax, L'entreprise et le droit, Paris, LGDJ, 1957, $\mathrm{n}^{\circ} 319$.

56. H. Temple, Les sociétés de fait, Paris, LGDJ, 1975, n² 226.

57. Y. GuYon, «Affectio societatis», JCl. Sociétés Traité, fasc. 20-10, n 41. 
d'intérêts inhérents au concubinage ${ }^{58} »$. Appliqué à la paroisse, ce raisonnement pousse à différencier chez les fidèles l'intention de collaborer et de vivre une aventure sociétaire, d'une part, et l'apostolat des laïcs prévu par l'Église et largement encouragé par le concile Vatican II ${ }^{59}$, d'autre part.

Quant à la preuve de la vocation aux bénéfices et aux pertes des fidèles, elle paraît absolument impossible à rapporter. Source de richesse spirituelle et humaine, la paroisse n'a aucunement vocation à permettre l'enrichissement financier de ses membres.

En conclusion, la paroisse, simple établissement de l'association diocésaine, occupe une place tout à fait singulière au sein de l'organisation religieuse catholique. Centrale pour chacun des fidèles, elle n'a pas d'autonomie juridique en droit civil et emprunte à la société plusieurs règles d'organisation et de fonctionnement afin de garantir une pastorale de qualité. Si le rapprochement entre le fonctionnement de la paroisse et celui de la société est possible, il n'a aucune influence sur la qualification de la paroisse en droit civil; elle ne saurait juridiquement risquer une requalification en société.

58. Cass. com., 3 avr. 2012, précit., contra Cass. com., 10 févr. 2015: Rev. sociétés 2015, p. 524 , note S. LacroiX-De SOUSA.

59. CONFÉRENCE des ÉVÊQUeS DE France, L'apostolat des laïcs: la libre association des fidèles en vue de l'apostolat: orientations pastorales, Paris, Bayard, 2000. 